\title{
Inhomogeneous assembly of driven nematic colloids ${ }^{\dagger}$
}

\author{
Josep M. Pagès, ${ }^{* a, b}$ Arthur V. Straube, ${ }^{b, c}$ Pietro Tierno, ${ }^{b, c}$ Jordi Ignés-Mullol, ${ }^{b, c}$ and \\ Francesc Sagués ${ }^{b, c}$ \\ We present a quantitative analysis of the nonequilibrium assembly of colloidal particles dispersed \\ in a nematic liquid crystal. The driven particles assemble into reconfigurable circular clusters by \\ liquid-crystal-enabled electrokinetic phenomena generated by an AC electric field that provides \\ propulsion along the local director. We identify the coexistence of different aggregation states, \\ including a central, jammed core, where short-range elastic attraction dominates, surrounded by \\ a liquid-like corona where particles retain their mobility but reach a mechanical equilibrium that \\ we rationalize in terms of a balance between centripetal phoretic drive and pairwise repulsion. An \\ analysis of the compressible liquid-like region reveals a linear density profile that can be tuned \\ with the field frequency, and a bond-orientational order that reaches a maximum at intermediate \\ packing densities, where elastic effects are minimized. Since the phoretic propulsion force acts \\ also on assembled particles, we compute the mechanical pressure and show that a hard-disk \\ equation of state can be used to describe the assembly of this driven system.
}

\section{Introduction}

Active colloidal suspensions are a flourishing research field in soft condensed matter, as they allow investigating the out-ofequilibrium physics of interacting organisms across different length scales ${ }^{1-3}$. The past few years have witnessed different experimental studies realized with either artificial ${ }^{4-10}$ or living units $^{11-14}$. From the point of view of theory and numerical simulations, attention has been mainly focused on collective dynamics aspects in interacting many-body systems ${ }^{1-3,15}$. In such cases, emerging effects are often difficult to predict based only on the knowledge of the behavior of the individual swimmers. In this respect, new physical concepts, like giant density fluctuations ${ }^{16-18}$, activity-driven phase separations ${ }^{19-21}$, active crystals ${ }^{9,22-24}$, and swarming phenomena ${ }^{8,17,25-30}$, have become by now hallmarks in this emergent research area.

In this article, we analyze the non-equilibrium assembly of interacting colloidal particles driven to form quasi-two-dimensional aggregates where phase coexistence emerges from the balance

\footnotetext{
a Departament de Ciència de Materials i Química Física, Universitat de Barcelona, Catalonia.

${ }^{b}$ Department of Mathematics and Computer Science, Freie Universität Berlin, Berlin, Germany.

${ }^{c}$ Departament de Física de la Matèria Condensada, Universitat de Barcelona, Catalonia.

${ }^{d}$ Institut de Nanociència i Nanotecnologia (IN $\left.{ }^{2} U B\right)$, Universitat de Barcelona, Catalonia.

a Universitat de Barcelona Institute of Complex Systems (UBICS), Universitat de Barcelona, Catalonia.

$\dagger$ Electronic Supplementary Information (ESI) available: video of a growing particle assembly. See DOI: 10.1039/cXsm00000x/
}

between phoretic propulsion and long-range pairwise repulsion. Unlike conventional studies where colloids are dispersed in isotropic media, our experiments are performed in an anisotropic nematic liquid crystal (LC), which provides control capabilities over the individual units ${ }^{31-35}$. In a recent work, we exploited the tunable nature of the LC medium to create reconfigurable topological defects that promote the formation of circular clusters and enable the collective transport of large ensembles of colloidal particles $^{33}$. For defects with perfect radial orientation, growing clusters reached configurations where different packing arrangements could be observed. Moreover, changing the strength of the phoretic drive led to a reversible modification of the packing density, suggesting the existence of a tunable pairwise repulsion that opposed the centripetal phoretic drive. In the current work, we present a detailed quantitative analysis of these experiments, both in terms of the density and the ordering of assembled colloidal particles, and provide evidences for the nature of the pairwise repulsion.

The structure of this paper is as follows. We begin by describing the experimental system and protocols in the next section. Then, we analyze the geometry of the colloidal assemblies in terms of their density distribution. Next, we analyze the bond-orientational order inside the assemblies, showing a nonmonotonic behavior with the distance from the center of the clusters. Finally, we characterize the liquid-like part of the aggregates in terms of an equation of state, after we compute the mechanical pressure exerted by the particles. We close with a discussion and some concluding remarks. 


\section{Materials and Methods}

\subsection{Preparation of the colloidal suspension}

We used polystyrene pear-shaped colloidal particles (Magsphere), $3 \times 4 \mu \mathrm{m}^{2}$ in size (width $\times$ length), which were dispersed in the nematic liquid crystal MLC7029 (Merk, $\varepsilon_{a}=-3.6$ at $\left.1 \mathrm{kHz}\right)^{33}$. The particles were received as an aqueous suspension, and were cleaned by centrifugation followed by ultrasonic resuspension of the precipitate in Milli-Q water. This process was repeated three times. Particles were dispersed in the liquid crystal by first drying $0.2 \mu \mathrm{L}$ of the $1 \% \mathrm{w} / \mathrm{v}$ aqueous suspension on a clean glass slide. A $10 \mu \mathrm{L}$ drop of the liquid was subsequently deposited on top of the dry particles, and gentle stirring was applied with a micropipette tip to favor particle dispersion.

\subsection{Preparation of the sample cells}

The mixture of colloids in liquid crystal was introduced by capillary action in the gap between two parallel glass plates that were glued together to prepare the experimental cell. About 20 $\mu \mathrm{m}$ plate separation was achieved using Mylar spacers (Goodfellow). The inner side of both plates featured an IndiumTin Oxide (ITO) thin film (Visiontek Systems, $100 \Omega \mathrm{sq}^{-1}$ ) that acted as the planar electrodes across which we applied an AC electric field. One of the ITO plates was spin-coated with the polyimide resin Nissan 0626, which promotes homeotropic (normal) anchoring of the LC molecules on the plate. Briefly, the resin was spin-coated at $2500 \mathrm{rpm}$ for 30s, subsequently dried at $80^{\circ} \mathrm{C}$ for 1 minute, and finally baked at $180^{\circ} \mathrm{C}$ for $1 \mathrm{~h}$. The other ITO surface was coated with a photosensitive selfassembled monolayer. In contrast to earlier protocols ${ }^{33}$, where we performed an in-situ reaction between a precursor aminosilane self-assembled monolayer and a carboxylic-acid azobenzene derivative, here we prepared a photosensitive azosilane monolayer in a single step. For this purpose, we employed the compound (E)-4-(4-((4-octylphenyl)diazenyl)phenoxy)- $N$-(3(triethoxysilyl)propyl)butanamide -henceforth called AZ-, which was custom-synthesized by GalChimia, Spain. AZ was combined at a ratio of 5:1 with (3-Aminopropyl)triethoxysilane (APTES, Sigma-Aldrich) to improve the system's photoresponse ${ }^{36}$. The deposition solution was prepared by dissolving the silanes and butylamine (Sigma-Aldrich; used as catalyzer ${ }^{37}$ ) in Toluene (99\%, Sigma-Aldrich) at a ratio 1:7:173. ITO-coated glass plates were first cleaned by sonication in a diluted solution of Micro-90 (Sigma-Aldrich), rinsed with Milli-Q water, dried with a stream of nitrogen and 30 minutes at $50^{\circ} \mathrm{C}$, and activated with $\mathrm{O}_{2}$ plasma before dipping them in the silane solution. The self-assembled monolayer was allowed to form for 120 minutes at $80^{\circ} \mathrm{C}$. Upon removal, the slides were quickly rinsed with toluene to avoid precipitation of the solute, followed by a 10-min wash with toluene under ultrasounds, and they where either readily used or stored under vacuum.

\subsection{Experimental setup}

A custom LED epi-illumination system incorporated in an upright polarizing optical microscope ${ }^{33}$ was used to both observe the sys- tem and control the local state of the AZ monolayer and, thus, the alignment of the LC director. In particular, the director aligns perpendicular to the plates in contact with the non-irradiated AZ layer and parallel to the plates in contact with the UV-irradiated AZ layer. Polarized brightfield imaging was performed with a red long-pass filter (Lambda, 645nm) to avoid perturbing the photosensitive layer. Irradiation was performed with a 365nm LED lamp (Thorlabs M365L2, $190 \mathrm{~mW}$ ), focused onto a spot of diameter $0.3 \mathrm{~mm}$ through a $20 \mathrm{x}$ microscope objective. An alternating current electric field was applied with a function generator (Agilent DSOX2002A) and a voltage amplifier (TREK PZD700).

Image processing was performed with ImageJ, and data analysis was conducted with Mathematica and IgorPro.

\section{Results}

\subsection{Density profile of the particle assemblies}

In absence of illumination or under white light, the AZ molecules adopt their elongated trans isomeric form, which leads to homeotropic anchoring of the LC molecules. Irradiating with UVlight $(360 \mathrm{~nm})$ induces, within seconds, the transition of the AZ molecules into the bent cis form, which triggers local planar anchoring of the $\mathrm{LC}^{33}$. The Gaussian intensity profile of the UV beam forces the LC molecules to develop an in-plane radial pattern starting from the center of the light spot*. The application of an AC electric field above the threshold for the in-plane reorientation of this LC with negative dielectric anisotropy leads to radial alignment of the director beyond the original light spot (Fig. 1). The anisometric inclusions align their symmetry axis with the local LC director because of the planar anchoring conditions on their surface and because of LC elasticity. In the frequency range $5-30 \mathrm{~Hz}$, electro-osmotic flows propel the particles along the local LC director, usually with the large lobule ahead ${ }^{\dagger}$ (Fig. 1a) ${ }^{33,34,38}$. In brief, the oscillating AC field generates convection rolls due to ion migration around the inclusions. Flow structure is determined by the LC director configuration around the particles and by the anisotropic ionic mobility. In the absence of perfect quadrupolar order, such as in the presence of anisometric inclusions (Fig. 1b), this process results in a net propulsion, since the fore-aft symmetry of the LC director around the particles is broken $^{34}$. Particles acquire a constant speed $v_{0}$, perpendicular to the electric field, moving within the viscous fluid. Therefore, a balance is established between viscous friction and the phoretic drive, so particles move as if propelled by a force $F=\gamma v_{0}$, where $\gamma$ is the friction coefficient of the particles. We have restricted our experiments to frequencies above $10 \mathrm{~Hz}$ to ensure a monotonic dependence of the particle speed (Fig. 1c), and we have kept the amplitude of the sinusoidal AC field at $0.76 \mathrm{~V} \mu \mathrm{m}^{-1}$. We have verified that the latter value is below the threshold for the onset of bulk electrohydrodynamic instabilities of the LC, which

\footnotetext{
* This configuration is preserved for more than 30 minutes when the UV source is switched off and the sample is observed under red light. ${ }^{36}$

$\dagger$ We have observed a certain dispersion in the particle orientation with respect to their direction of motion, attributable to the surface boojum defects being pinned away from the symmetry axis. ${ }^{43}$
} 

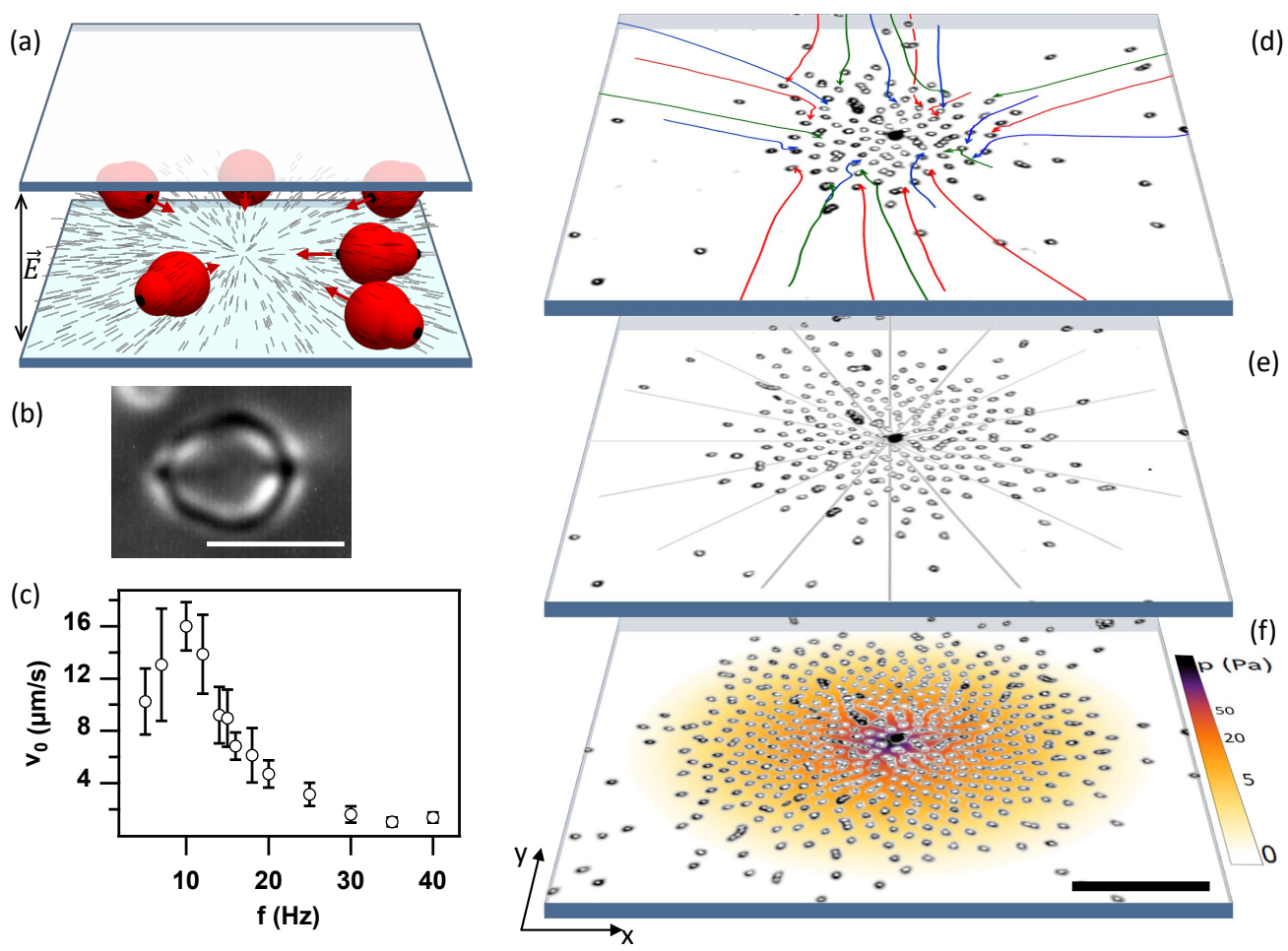

Fig. 1 (a) Sketch of the experimental setup. Anisometric colloidal particles dispersed in a nematic LC are propelled via application of an AC electric field normal to the sample plane. Reconfigurable boundary conditions on one of the two bounding plates allow to define "attractor points" in the LC cell to assemble the particles. (b) Micrograph of a $10 \times 8 \mu \mathrm{m}^{2}$ pear-shaped particle displaying the two surface boojum defects. The scale bar is $10 \mu \mathrm{m}$. (c) Mean velocity of individual particles, $v_{0}$, as a function of the driving frequency for an electric field amplitude $0.76 \mathrm{~V} \mu \mathrm{m}^{-1}$. Error bars are the standard deviation over 10 particles. (d)-(f) Micrographs of a quasi-two-dimensional cluster of particles growing under a $f=20 \mathrm{~Hz} \mathrm{AC}$ field with $200 \mathrm{~s}$ of total elapsed time. In (d), the trajectories of a few particles that join the cluster are superimposed to the image. The radial director field lines that converge into a central topological defect are depicted in (e). The color map in panel (f) represents the computed mechanical pressure within the cluster (see text). The scale bar in (f), for images (d)-(f), is $50 \mu \mathrm{m}$.

we want to avoid ${ }^{39 \ddagger}$. Moreover, the field amplitude acts as a scaling parameter both in the phoretic drive and in the inter-particle interactions. In contrast, as shown below, the AC frequency has a more subtle influence in the relevant physical processes leading to the present colloidal assembly process.

Once the LC director is in a radial configuration (Fig. 1(d)(f)), the AC field drives the particles within an area of influence of several square millimeters towards the topological defect created at the center of the original light spot (Fig. 1(d)). The far field trajectories of individual particles are radial and centripetal ${ }^{\S}$, following the LC director (Fig. 1(d,e)). As more particles arrive, the nematic colloids assemble into a circular cluster with a steady-state inter-particle distance comparable to the size of the inclusions. Near the cluster, trajectories depart from the radial direction, until particles eventually accommodate into the arrested cluster. The average inter-particle distance changes as the cluster grows, becoming smaller close to the center than near the clus-

$\ddagger$ Although the amplitude affords a well-known quadratic influence on the particle speed $^{32}$, the need to avoid the onset of instabilities in the LC limits the range of usable field amplitudes.

$\S$ Trajectories can also be centrifugal, but such particles escape or never enter the field of view. ter periphery (Fig. 1(e,f)). This behavior suggests the existence of a net repulsive pairwise interaction that balances the steady phoretic drive acting on individual particles and preventing their irreversible aggregation induced by LC elasticity 40,41 . The repulsive interaction among inner particles has to balance the phoretic drive of an increasing number of arriving outer particles, which explains the inhomogeneous density distribution.

In Fig. 2(a), we show an aggregate after a few hundred particles have accumulated. The cluster organizes in three welldefined regions characterized by different states of aggregation. The innermost core (region I in Fig. 2(a)) is an arrested, jammed state that features only minor particle rearrangements as the assembly grows. In Fig. 2(b), we monitor the density profile at different stages during colloidal assembly. We observe that the arrested core starts to be visible when the cluster achieves a minimum radius of approximately $80 \mu \mathrm{m}$ under the conditions of this experimental realization. Within the core, the radial-averaged particle density is roughly homogeneous, although far from closepacking. We clearly observe short-range heterogeneity in the form of particle chaining (Fig. 2(a)), presumably due to the LCmediated anisotropic interactions ${ }^{40,41}$. The size of the core keeps growing with the arrival of new particles, but its packing density remains nearly unchanged (Fig. 2(b)). 
(a)

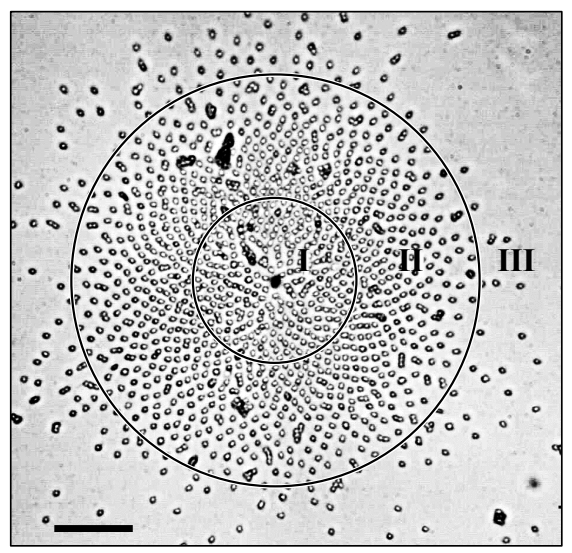

(b)

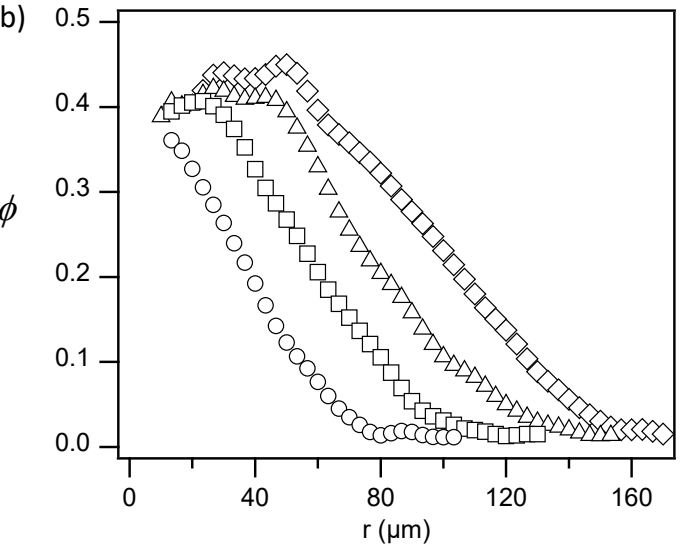

Fig. 2 Growing colloidal assembly. (a) Micrograph of a cluster assembling while a $f=20 \mathrm{~Hz}$ AC field is being applied. The scale bar is $50 \mu \mathrm{m}$ (see also Video S1). Numbers indicate the three regions discussed in the text. (b) Area fraction occupied by the particles $v s$ distance from the cluster center for the same experiment. Elapsed times since the beginning of assembly are $150 \mathrm{~s}(\bigcirc)$, 250s $(\square)$, 350s $(\triangle)$, and 500s $(\diamond)$.

The intermediate region (II) features a liquid-like state, where particles retain their motility and appear clearly separated under the microscope ${ }^{\top}$. The radial-averaged particle area fraction, $\phi(r)$, decreases roughly linearly with the distance to the boundary with region (I) (Fig. 2(b)). As the cluster grows, both the slope and the radial span of this region remain roughly unchanged. In other words, clusters of different sizes feature the same radial width and structure of the liquid-like corona, and they are only different in the growing size of the inner arrested core.

When comparing assemblies formed at different driving frequencies, clusters feature a similar packing density in the arrested cores (region I) but are clearly different in terms of the width and density distribution of region (II) (Fig. 3(a)). We have compared the average slope in the range of linear density profile for experiments performed at different frequencies (Fig. 3(b)), revealing a nonlinear trend with the driving frequency. Remarkably, our data suggests that the slope is, in fact, proportional to the corresponding phoretic speed of the driven isolated particles (see inset in

I Some aggregated particles are observed, but these were present before the phoretic drive was enabled.
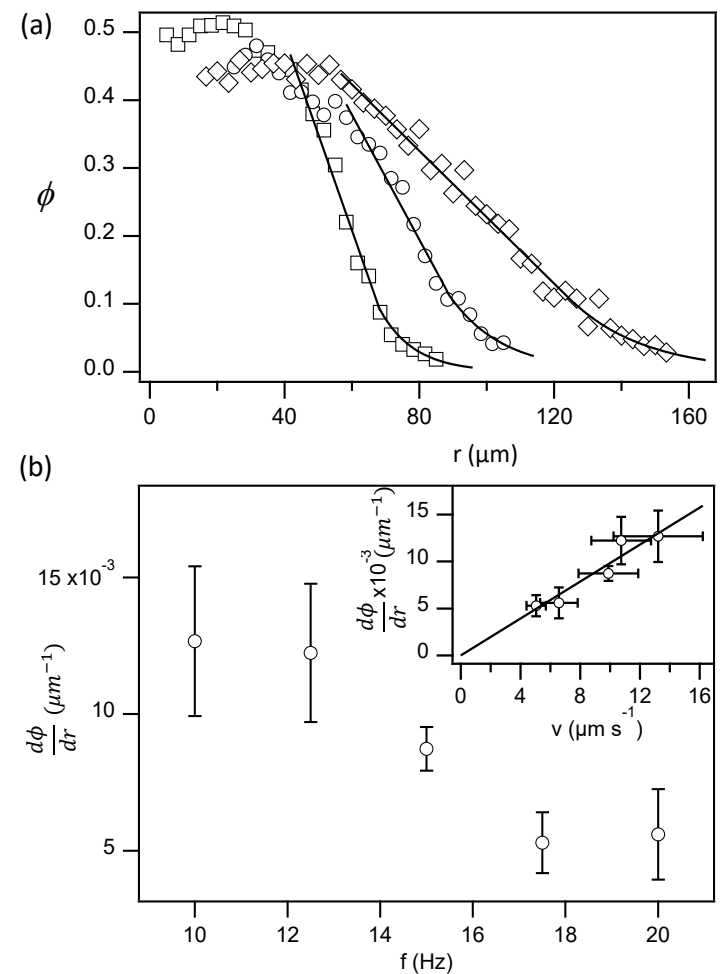

Fig. 3 Analysis of the experimental colloidal assemblies. (a) Area fraction occupied by the particles $v s$ distance from the cluster center for experiments at $f=10(\square), 15(\bigcirc)$, and $20(\diamond) \mathrm{Hz}$. The line through the data is a linear fit to the area fraction profile in region (II), and an exponential fit in the dilute region (III). (b) Average slope of the density profile in region (II), $v s$ frequency. In the inset, the same data is plotted as a function of $v_{0}$. The error bars are the standard deviations for different realizations.

Fig. 3b).

Finally, at area fractions below 0.1 (region III in Fig. 2(a)), $\phi(r)$ follows, to a good approximation, an exponential decay to zero, in analogy to an ideal gas under barometric conditions, and similarly to the reported behavior of sedimenting active colloidal particles in very diluted regimes ${ }^{42}$ (see below).

\subsection{Scaling arguments for the density profile}

The linear spatial variation and the scaling with the particle speed $v_{0}$ of the density profile in region II (Fig. 3) can be qualitatively understood with the help of a simple toy model (Fig. 4). Let us consider a one-dimensional stack of $N$ particles in mechanical equilibrium, with a constant force $f$ pulling each particle towards an immobilized particle to the left, and a pairwise repulsion that we can model as a spring with constant $K$ and length $q$ between neighboring particles.

When the left-most particle is pinned, force balance leads to an equilibrium particle position for the $n^{\text {th }}$ particle

$$
r_{n}=n q-n\left[N-\frac{n-1}{2}\right] \ell,
$$

where $\ell=f / K$.

We can then estimate the one-dimensional particle number density as $n\left(r_{n}\right) \simeq 1 / \Delta r_{n}$. Using Eq. 1 , and in the limit $N \gg 1$, 


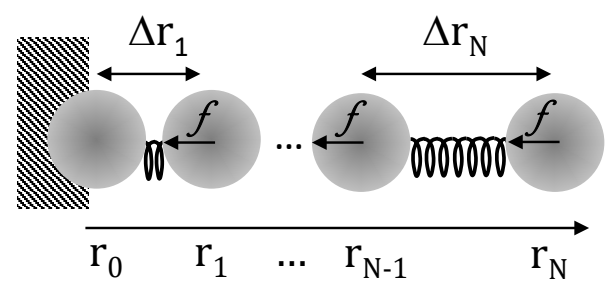

Fig. 4 Toy model system for a 1-d equilibrium assembly of particles individually propelled by a force $f$ that is balanced by a nearest-neighbor repulsion potential. The pairwise repulsion between nearest neighbors is represented by a spring, as shown between the two outermost particles.

we find

$$
n(r) \simeq \frac{n_{*}}{\sqrt{1+2 n_{*}^{2} \ell r}} .
$$

Here, $n_{*}=(q-N \ell)^{-1}$ is the maximum number density occurring between the first and second particles, where interparticle distance is shortest. For small spring distortions, Eq. 2 can be approximated as

$$
n(r) \approx n_{*}-\frac{n_{*}^{3} f}{K} r,
$$

resulting in a linear decay, consistently with our experimental observations. Moreover, assuming that the force acting on each particle in the assembly is the same that sets individual particles into constant motion, $f=\gamma v_{0}$, we recover the linear scaling with $v_{0}$ of the density decay. In Eq. 3, the spring constant $K$ will be related to the pairwise particle interaction that, in principle, one would expect to depend on the driving frequency. Remarkably, our experiments suggest that such dependence is relatively weak.

\subsection{Bond-orientational order}

Particle ordering in this quasi-two-dimensional assembly can be further characterized using a radially-resolved bond-orientational order parameter that quantifies how close to a hexagonal arrangement the neighborhood of each particle is. For this purpose, we compute, for each particle, the parameter

$$
\psi_{6, k}=\left|\frac{1}{N_{k}} \sum_{j=1}^{N_{k}} \exp \left(-i 6 \theta_{k, j}\right)\right|^{2} .
$$

Here, the sum extends over the $N_{k}$ nearest neighbors of particle $k$ in the assembly, and $\theta_{k, j}$ is the bond-orientational angle between the particle and its $j$ th neighbor. As an approximation for our slightly non-spherical colloids, we set the nodes of the lattice at the center of mass of each particle. Nearest neighbors were determined by the Delaunay triangulation of the particle positions in the assembly using Mathematica (Figure 5). For a perfectly hexagonal arrangement, this definition would result in $\psi_{6, k}=1$. The radially-resolved order parameter, $\psi_{6}(r)$ is further obtained by circularly averaging $\psi_{6, k}$ (Figure $5(\mathrm{c})$ ).

In Fig. 5(c) we monitor the average radial profile $\psi_{6}$ at different stages during the formation of a particle assembly. The value (a)

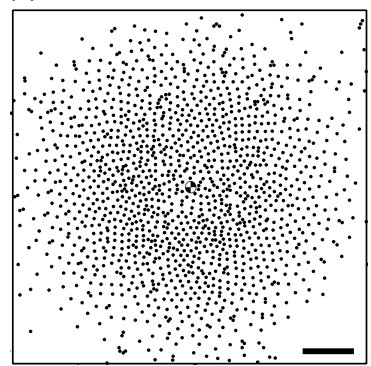

(c)

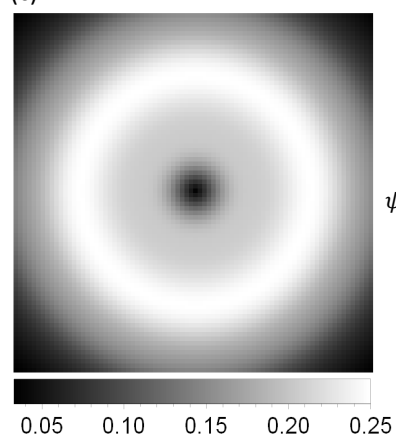

(b)

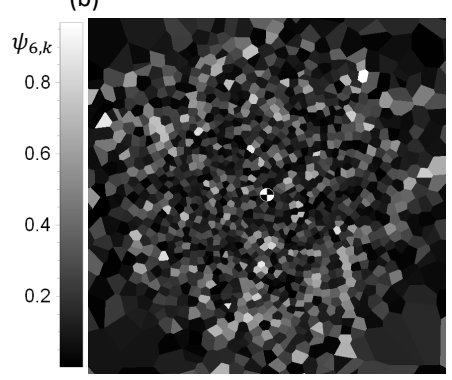

(d)

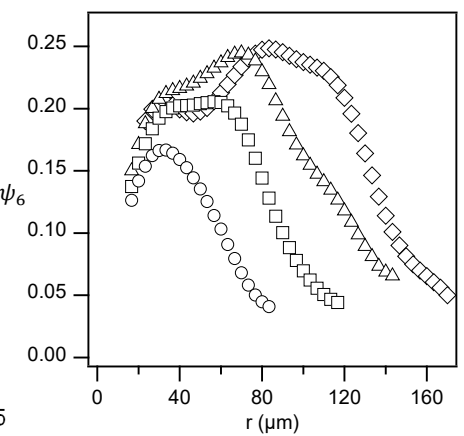

Fig. 5 (a) Tracked particle positions after an elapsed time of 350s for the same experiment shown in Fig. 2a. The scale bar is $50 \mu \mathrm{m}$. (b) Delaunay triangulation of the particle assembly and associated value of the bond-orientational order parameter, $\psi_{6, k}$. (c) Distribution of the circularlyaveraged bond-orientational order parameter, $\psi_{6}(r)$ for the same data. (d) Profile of the bond-orientational order parameter as a function of the distance from the cluster center. Elapsed times since the beginning of assembly are 150s $(\bigcirc)$, 250s $(\square)$, 350s $(\triangle)$, and 500s $(\diamond)$.

of $\psi_{6}$ in region (I) is lower than expected for a solid-like system where particles are close-packed. Disorder in the present system is due to the use of non-spherical particles, particle polydispersity, or surface asperities that may change particle orientation with respect to the local director field ${ }^{43}$. Another important effect is the short-range elastic interactions mediated by the LC matrix, that promotes the off-centered chaining of the particles and prevents optimal packing. Interestingly, we find that the average value of $\psi_{6}$ changes in a non-monotonic way with the distance from the cluster center, achieving a maximum value in region (II), where the density profile decays linearly, and further dropping to zero in region (III). The position of this maximum shifts to higher radii as the cluster grows, consistently with the growth process described above, where cluster evolves by increasing the size of region (I), and preserving the thickness and particle organization of region (II). We showed above that this is true for the linear density profile, and here we have shown that this is also true for the presence of a local maximum in the bond-orientational order. The observed non-monotonic behavior of $\psi_{6}$ is in contrast to experiments with sedimenting spherical colloidal particles, where the hexagonal order is a monotonic function of the packing density ${ }^{44}$. The unusual behavior reported here can be attributed to the existence of long range particle repulsion, which enhances hexagonal ordering in the lower-density region, where the short-range particle chaining, dominant in region I, is absent. 


\section{Nonequilibrium equation of state}

As discussed above, the density profile within the particle assemblies is the result of a balance between the centripetal phoretic drive acting on individual particles, and the long range pairwise repulsion. Similar assemblies, albeit in linear geometry, have been studied during the slow gravitational sedimentation of charged colloidal particles ${ }^{45}$, Brownian particles ${ }^{44}$, and active particles ${ }^{42,44}$. In these systems, the mechanical pressure exerted inside the assembly was readily calculated from the force exerted by outer particles, and resulted in a measurable equation of state that related packing density with local pressure.

We have performed a similar analysis in our system, which relies on the hypothesis that the contribution of individual particles to the pressure in the assembly results from the same force that drives isolated particles at constant speed within the viscous fluid (Fig. 1(b)). As discussed above, particle propulsion results from unbalanced electrokinetic flows. When particles are assembled within regions (II) and (III), interparticle distance is of the order, or larger than particle size. It is thus reasonable to assume that the electrokinetic flows that surround each particle are not very different from those around a free particle, and thus the particle will contribute to the pressure inside the assembly with a centripetal force $F=\gamma v_{0}$. This assumption is likely to be invalid in region (I), where particle chaining dominates the assembly and electrokinetic flows should be perturbed. Actually, the observation that the average packing density is uniform in region (I) even though density is far from close-packing, suggests that the phoretic drive acting on individual particles is quite different from that on free particles.

With these assumptions, we can compute the mechanical pressure $p$ within the fluid regions of an assembly at, a given distance $r$ from the cluster center, as

$$
p(r)=\frac{F}{w r} \int_{r}^{\infty} \frac{\phi\left(r^{\prime}\right)}{a_{0}} r^{\prime} d r^{\prime} .
$$

Here, $w$ is the thickness of the particle layer, which we take as the diameter of the largest particle lobule, $w=3 \mu \mathrm{m}, a_{0}$ is the average area footprint of our particles, and we consider $p=0$ outside the cluster.

In order to quantify $F$ from the measurement of $v_{0}$ we consider, as a first approximation, the friction coefficient of a spherical particle, $\gamma=6 \pi \eta_{\|} a$. We use, as hydrodynamic radius, $a=1.5 \mu \mathrm{m}$, which is the radius of the largest lobule in our particles. Although the LC is an anisotropic fluid, characterized by the viscosities $\eta_{\|}$ and $\eta_{\perp}$ depending on whether the flow is parallel or perpendicular to the director $\hat{\boldsymbol{n}}$, particle motion in our system always takes place along $\hat{\boldsymbol{n}}$, so only $\eta_{\|}$is relevant. We have estimated a value $\eta_{\|}=1.4 \times 10^{-3} \mathrm{~Pa} \cdot \mathrm{s}$ by comparing, in a separate experiment, the motion of paramagnetic colloidal particles propelled by an external magnetic field gradient both in water and in the aligned LC.

The results of this data analysis is presented in Fig. 6 as pressure vs. area fraction isotherms. We have combined data from experiments performed at 10 and $20 \mathrm{~Hz}$, for which free-particle speeds, and thus exerted centripetal forces, differ by a factor of four. We observe that both sets of data overlap into a single

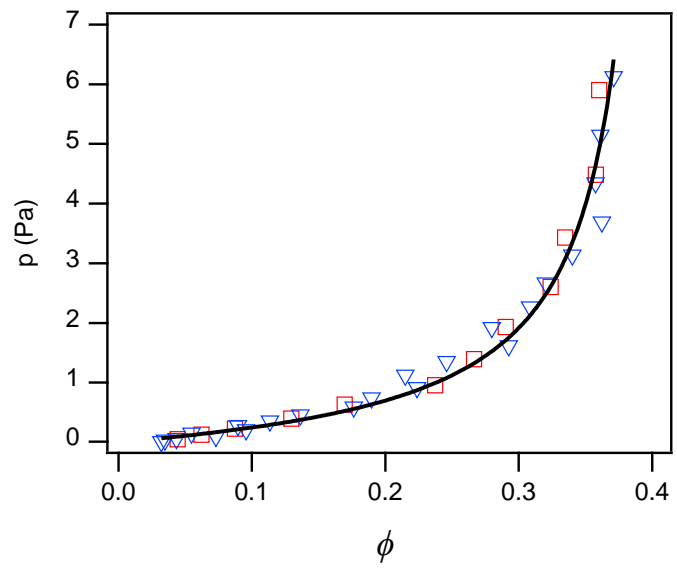

Fig. 6 Estimated mechanical pressure as a function of the particle area fraction in an assembled cluster for experiments at $10 \mathrm{~Hz}(\square)$ and $20 \mathrm{~Hz}$ $(\nabla)$. The solid curve is a fit to a hard-disk equation of state in the compressible, liquid-like region (see text).

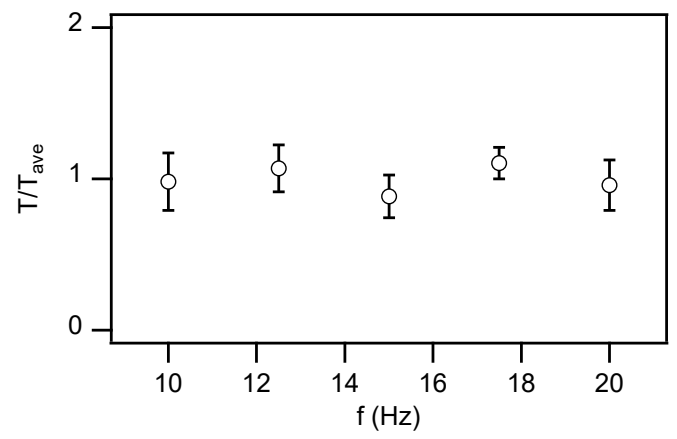

Fig. 7 Effective temperature relative to its average value, obtained from the exponential fit of the density profile in region (III) at different driving frequencies (see text). The error bars are the standard deviations for different realizations.

isotherm suggesting that pairwise interactions are the same for the studied range of driving frequencies. Because of finite size effects related to the radial geometry of the patterns, $p(r)$ will not only depend on the particle density profile but also on the radius of curvature of the assembly. This has been taken into account when comparing data of different experiments in Fig. 6, choosing ensembles where the onsets of the linear density profiles occur at similar distances form the cluster center. Although we cannot reliably compute the pressure inside region (I), the continuity of the measured density profiles suggests that the pressure-density isotherms in our system will not feature a plateau, unlike what is reported for slowly sedimenting spherical colloids in isotropic fluids ${ }^{44,45}$.

The observed trend for the $p$ - $\phi$ data in region II of the colloidal assemblies can be well described by an equation of state similar to the hard-disk case ${ }^{46}$,

$$
p \propto \frac{\phi}{1-2 \phi+\left(\phi_{0}^{2}-1\right)\left(\frac{\phi}{\phi_{0}}\right)^{2}},
$$


where $\phi_{0}$ is the extrapolated close-packing particle area fraction. Such behavior may be understood by considering that, under the diluted conditions of region II, the large interparticle distances prevent the LC-mediated attractive anisotropic interactions to set in, thus rendering the colloidal behavior effectively similar to a hard-sphere liquid.

Unlike other aggregation experiments reported with active ${ }^{42}$ or driven passive diffusive micro-particles ${ }^{44}$, thermal fluctuations are negligible in our system, given the relatively large viscosity of the dispersing medium. To reinforce this idea, we have estimated, for the gas-like region III, an effective temperature by fitting $\phi(r) \propto \exp \left(-\gamma v_{0} r /\left(k_{B} T_{\text {eff }}\right)\right)$. As shown in Fig. 7, we find that $T_{\text {eff }}$ is frequency-independent, consistently with the athermal nature of our system, and different from systems of active particles $^{42}$.

\section{Discussion}

The reported experiments analyze a process of nonequilibrium colloidal assembly mediated by a nematic liquid crystal. Unlike their equilibrium counterparts, where elastic interactions determine the structure of the assemblies, in the present case elasticity plays a secondary role, and is only determinant when particles are closely packed, in region I. Strictly speaking, elasticity also plays a role in forcing the driven particles to orient along the local LC director, thus steering the colloids that are indeed propelled by the out-of-equilibrium electrokinetic effects. In this respect, our colloidal particles are driven rather than active, since their moving direction is externally set.

The steady-state particle arrangement we have reported in regions (II) and (III) also illustrates the non-equilibrium nature of our system. Removing the phoretic drive by increasing the AC frequency has a significant impact in those regions, since both the centripetal propulsion and long-range pairwise repulsion are removed simultaneously. Particles in the assembly drift freely, with the eventual chain formation due to short-range elastic attraction, which is frequency-independent.

Our experiments provide valuable information to understand the nature of the involved physical effects that lead to pairwise interaction. At short range, LC-mediated elasticity dominates, as evidenced by the chaining inside the arrested core. On the other hand, the same induced flows that propel individual particles will contribute to the long-range pairwise repulsion when convection rolls surrounding two neighboring particles overlap. Nevertheless, the observed scaling of $\phi(r)$ with $v_{0}$ in region II (Fig. 3(b)) reinforces the assumption that other frequency-dependent physical ingredients are necessary to account for the long-range repulsion, since the resulting effective pairwise interaction appears to be frequency independent (Sec. 3.2).

One likely candidate to contribute to this pairwise repulsion is dipolar interaction, which has recently been proven consistent with the dynamics of swarms ${ }^{47}$, since particles will acquire an induced out-of-plane electric dipole, equally oriented for all particles. In order to confirm the importance of dipolar interactions, we performed additional experiments in different configurations. In the example shown in Fig. 8(a)-(c) dipolar attraction dominates when the phoretic velocity is parallel to $\boldsymbol{E}$, which hap- pens for LC with positive dielectric anisotropy. In that case, the in-plane dipoles promote particle chaining and aggregation into compact clusters. In the experiments reported above, however, particles are coplanar, and the induced dipoles are perpendicular to the plane of the sample. This results in a net pairwise repulsion that contributes to cluster stability.

If we analyze the geometry of our assemblies, in particular focusing on the bond-orientational order, we find significant differences when compared to previous literature that involves localized active colloidal assembly $9,23,48-52$. In those works, one finds typically a transition between close-packed and dispersed phases, rather than the richness of aggregation regimes reported here. This is likely due to the presence of long-range pairwise repulsion featured by our system, balanced by the centripetal drive. Unlike earlier works with spherical particles, the shape of our colloids coupled with the LC elasticity prevent their efficient close packing. This has a clear impact in the structure of region I, where the maximum area fraction is well below what can be achieved with spherical particles. We argue, however, that these effects are subdominant in the liquid-like region II, where particles are not in close contact, and long-range pairwise repulsion dominates.

Our aggregation process bears similarities to earlier onedimensional colloidal sedimentation experiments, in particular to those featuring interparticle repulsion 44,45 . In that context, the monotonous decrease of bond-orientational order from its maximum value in the close-packed assembly was reported, evidencing a transition from hexagonal to hexatic order ${ }^{44}$. Differently, a peculiarity of our system is the non-monotonous trend of the bond-orientational order, achieving a local maximum in the region where area fraction is around 0.25 , i.e., well within region II (Fig. 5), where short-range elastic attraction effects are negligible.

Finally, we have analyzed the instantaneous configuration of the assemblies in terms of a mechanical force balance between centripetal drive acting on individual particles and pairwise repulsion that opposes clustering. It is interesting to observe that the core of the assembly is far from close packed, yet it constitutes a jammed percolating network that can withstand the force exerted by outer particles. Packing in this region might be altered by changing the geometry of the underlying LC director from radial to spiral, which adds an azimuthal component to the local phoretic drive, as we reported in earlier experiments ${ }^{33}$.

\section{Concluding remarks}

In summary, we have investigated the out-of-equilibrium twodimensional assembly of anisometric colloidal particles driven toward a reconfigurable topological defect in a nematic liquid crystal. The anisotropy of the dispersing medium combined with the geometry of the experiments result in the onset of different electrokinetic phenomena generated by the alternating current electric field. The latter provides a constant propulsion local to each particle that promotes aggregation when coupled to the radial LC director, but it also generates a pairwise repulsion that leads to a steady state assembly characterized by different aggregation phases and packing densities.

The reported analysis has mostly focused in the liquid-like part 

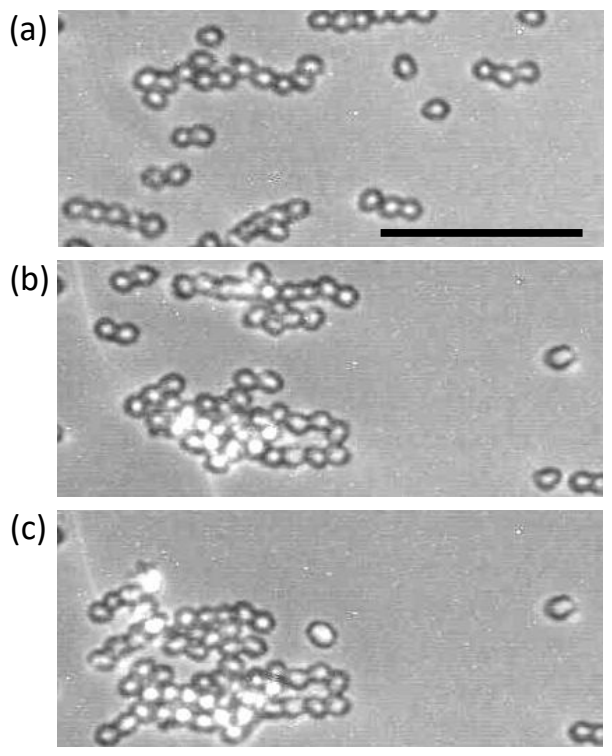

Fig. 8 (a)-(c)Pear-shaped particles $\left(8 \times 10 \mu \mathrm{m}^{2}\right)$ embedded in a LC with positive dielectric anisotropy (5CB) and driven by an in-plane AC field of frequency $10 \mathrm{~Hz}$ and amplitude $0.1 \mathrm{~V} / \mu \mathrm{m}$ (horizontal in the snapshots). The scale bar is $50 \mu \mathrm{m}$.

of the aggregates, where particles reach mechanical equilibrium without close contact. Within this phase, we find a reentrant behavior in the colloidal ordering, as the bond-orientational order parameter reaches a local maximum when short-range elastic effects can be neglected. By assuming that the phoretic drive acting on isolated particles is preserved within this phase, we have computed the mechanical pressure within the assembly, and have found that this hypothesis is consistent when comparing pressuredensity isotherms obtained with different phoretic drives.

Our results suggest that pairwise repulsion is a combination of multiple physical effects, including hydrodynamic coupling of the electrokinetic flows that surround individual colloidal particles and dipolar repulsion enabled by our choice of material and experimental parameters. Importantly, the elasticity of the liquid crystal plays only a secondary role outside of the arrested core of the colloidal clusters. Although we have imposed the formation of radial assemblies, we do not expect our results to depend on the choice of geometry, and similar effects should be observed in defect-free assembly. On the whole, our experiments demonstrate enhanced control capabilities for microscale colloidal assembly endowed by their dispersion in anisotropic solvents.

\section{Acknowledgements}

We thank A. Ortiz-Ambriz for assistance in measuring the LC viscosity. The authors acknowledge fruitful discussions with L. Schimansky-Geier and I. Sokolov. J. M. P. acknowledges funding from the European Union's Horizon 2020 Fetopen "AbioMatter", grant No. 665440. Experiments were funded by MINECO (AEI/FEDER, EU), projects FIS 2013-41144P and FIS2016-78507-C2-1-P . P.T. acknowledges support from the European Research Council grant agreement No. 335040, from
MINECO ( AEI/FEDER, EU), project FIS2016-78507-C2-2-P, and DURSI, project 2014-SGR-878. A. V. S. acknowledges partial support from the Excellence Initiative and SFB1114 of the German Research Foundation.

\section{References}

1 C. Bechinger, R. Di Leonardo, H. Löwen, C. Reichhardt, G. Volpe and G. Volpe, Reviews of Modern Physics, 2016, 88, year.

2 A. Zöttl and H. Stark, Journal of Physics: Condensed Matter, 2016, 28, year.

3 J. Elgeti, R. G. Winkler and G. Gompper, Rep Prog Phys, 2015, 78, 056601.

4 W. F. Paxton, K. C. Kistler, C. C. Olmeda, A. Sen, S. K. St Angelo, Y. Cao, T. E. Mallouk, P. E. Lammert and V. H. Crespi, $J$ Am Chem Soc, 2004, 126, 13424-31.

5 J. Howse, R. Jones, A. Ryan, T. Gough, R. Vafabakhsh and R. Golestanian, Physical Review Letters, 2007, 99, 048102.

6 H.-R. Jiang, N. Yoshinaga and M. Sano, Physical Review Letters, 2010, 105, 268302.

7 S. Sanchez, A. N. Ananth, V. M. Fomin, M. Viehrig and O. G. Schmidt, J Am Chem Soc, 2011, 133, 14860-3.

8 S. Thutupalli, R. Seemann and S. Herminghaus, New Journal of Physics, 2011, 13, year.

9 J. Palacci, S. Sacanna, A. P. Steinberg, D. J. Pine and P. M. Chaikin, Science, 2013, 339, 936-40.

10 J. Li, V. V. Singh, S. Sattayasamitsathit, J. Orozco, K. Kaufmann, R. Dong, W. Gao, B. Jurado-Sanchez, Y. Fedorak and J. Wang, ACS Nano, 2014, 8, 11118-25.

11 C. Dombrowski, L. Cisneros, S. Chatkaew, R. E. Goldstein and J. O. Kessler, Phys Rev Lett, 2004, 93, 098103.

12 H. P. Zhang, A. Be'er, E. L. Florin and H. L. Swinney, Proc Natl Acad Sci U S A, 2010, 107, 13626-30.

13 T. Sanchez, D. T. Chen, S. J. DeCamp, M. Heymann and Z. Dogic, Nature, 2012, 491, 431-4.

14 P. Guillamat, J. Ignes-Mullol and F. Sagues, Proc Natl Acad Sci U S A, 2016, 113, 5498-502.

15 M. C. Marchetti, J. F. Joanny, S. Ramaswamy, T. B. Liverpool, J. Prost, M. Rao and R. A. Simha, Reviews of Modern Physics, 2013, 85, 1143-1189.

16 R. Aditi Simha and S. Ramaswamy, Phys Rev Lett, 2002, 89, 058101.

17 J. Toner, Y. Tu and S. Ramaswamy, Annals of Physics, 2005, 318, 170-244.

18 V. Narayan, S. Ramaswamy and N. Menon, Science, 2007, 317, 105-8.

19 G. S. Redner, M. F. Hagan and A. Baskaran, Phys Rev Lett, 2013, 110, 055701.

20 M. E. Cates and J. Tailleur, Annual Review of Condensed Matter Physics, 2015, 6, 219-244.

21 M. C. Marchetti, Y. Fily, S. Henkes, A. Patch and D. Yllanes, Current Opinion in Colloid and Interface Science, 2016, 21, 34 43.

22 B. M. Mognetti, A. Saric, S. Angioletti-Uberti, A. Cacciuto, 
C. Valeriani and D. Frenkel, Phys Rev Lett, 2013, 111, 245702.

23 I. Buttinoni, J. Bialké, F. Kümmel, H. Löwen, C. Bechinger and T. Speck, Physical Review Letters, 2013, 110, 238301.

24 O. Pohl and H. Stark, Eur Phys J E Soft Matter, 2015, 38, 93.

25 T. Vicsek, A. Czirok, E. Ben-Jacob, I. I. Cohen and O. Shochet, Phys Rev Lett, 1995, 75, 1226-1229.

26 F. Schweitzer, Brownian agents and active particles : collective dynamics in the natural and social sciences, Springer, Berlin ; New York, 2003.

27 F. Peruani, A. Deutsch and M. Bar, Phys Rev E Stat Nonlin Soft Matter Phys, 2006, 74, 030904.

28 H. Chate, F. Ginelli, G. Gregoire and F. Raynaud, Phys Rev E Stat Nonlin Soft Matter Phys, 2008, 77, 046113.

29 A. Bricard, J. B. Caussin, N. Desreumaux, O. Dauchot and D. Bartolo, Nature, 2013, 503, 95-8.

30 S. Saha, R. Golestanian and S. Ramaswamy, Phys Rev E Stat Nonlin Soft Matter Phys, 2014, 89, 062316.

31 O. D. Lavrentovich, Current Opinion in Colloid and Interface Science, 2016, 21, 97-109.

32 S. Hernàndez-Navarro, P. Tierno, J. Ignes-Mullol and F. Sagues, Soft Matter, 2013, 9, 7999-8004.

33 S. Hernandez-Navarro, P. Tierno, J. A. Farrera, J. Ignes-Mullol and F. Sagues, Angew Chem Int Ed Engl, 2014, 53, 10696-700.

34 I. Lazo, C. Peng, J. Xiang, S. V. Shiyanovskii and O. D. Lavrentovich, Nat Commun, 2014, 5, 5033.

35 S. Zhou, A. Sokolov, O. D. Lavrentovich and I. S. Aranson, Proc Natl Acad Sci U S A, 2014, 111, 1265-70.

36 P. Oswald and J. Ignes-Mullol, Physical Review E, 2017, 96, 032704.

37 D. Walba, C. Liberko, E. Korblova, M. Farrow, T. Furtak, B. Chow, D. Schwartz, A. Freeman, K. Douglas, S. Williams, A. Klittnick and N. Clark, Liquid Crystals, 2004, 31, 481-489.

38 O. D. Lavrentovich, I. Lazo and O. P. Pishnyak, Nature, 2010, 467, 947-50.

39 P. Oswald and P. Pieranski, Nematic and cholesteric liquid crystals : concepts and physical properties illustrated by experiments, Taylor \& Francis, Boca Raton, 2005.

40 I. Smalyukh, O. Lavrentovich, A. Kuzmin, A. Kachynski and P. Prasad, Physical Review Letters, 2005, 95, 157801.

41 I. Muševič, Liquid Crystal Colloids, 2017.

42 F. Ginot, I. Theurkauff, D. Levis, C. Ybert, L. Bocquet, L. Berthier and C. Cottin-Bizonne, Physical Review X, 2015, 5, 011004.

43 S. Hernàndez-Navarro, P. Tierno, J. Ignés-Mullol and F. Sagués, Molecular Crystals and Liquid Crystals, 2015, 610, 163-172.

44 A. L. Thorneywork, J. L. Abbott, D. Aarts and R. P. A. Dullens, Phys Rev Lett, 2017, 118, 158001.

45 M. A. Rutgers, J. H. Dunsmuir, J. Z. Xue, W. B. Russel and P. M. Chaikin, Physical Review B, 1996, 53, 5043-5046.

46 A. Santos, M. López de Haro and S. Bravo Yuste, The Journal of Chemical Physics, 1995, 103, 4622-4625.

47 A. V. Straube, J. M. Pagès, A. Ortiz-Ambriz, P. Tierno, J. IgnésMullol and F. Sagués, New Journal of Physics, 2018, 20,
075006.

48 I. Theurkauff, C. Cottin-Bizonne, J. Palacci, C. Ybert and L. Bocquet, Phys Rev Lett, 2012, 108, 268303.

49 J. Yan, M. Bloom, S. C. Bae, E. Luijten and S. Granick, Nature, 2012, 491, 578-81.

50 J. Yan, M. Han, J. Zhang, C. Xu, E. Luijten and S. Granick, Nat Mater, 2016.

51 M. Han, J. Yan, S. Granick and E. Luijten, Proc Natl Acad Sci U S A, 2017, 114, 7513-7518.

52 A. Aubret, M. Youssef, S. Sacanna and J. Palacci, Nature Physics, 2018. 


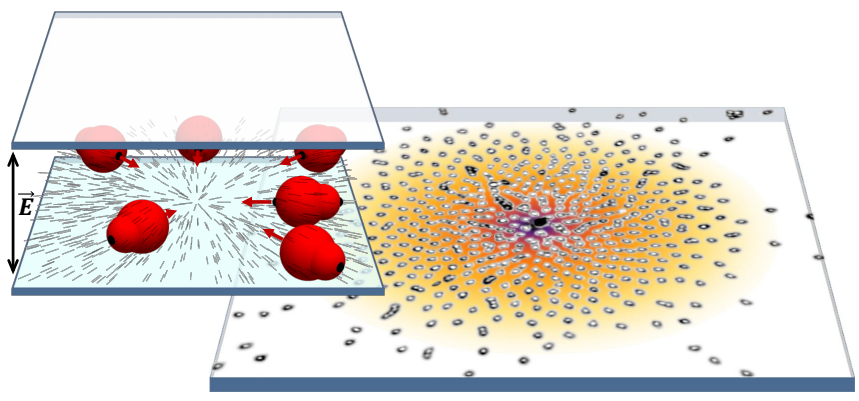

TOC picture. Colloidal particles are assembled into different states of aggregation driven by liquid-crystal-enabled electrokinetic propulsion. 